\title{
Study on Methods for Fish-eye Image Correction Based on Spherical Projection Model
}

\author{
Yicong Zhang, Shujun Zhou \\ Zhuhai College of Jilin University, Zhuhai, 519040, China \\ 8176156@qq.com
}

Keywords: Fish-eye, image, correction.

\begin{abstract}
For short focal length and large field of view, fish-eye lens has been widely applied in such fields as virtual reality, video surveillance, intelligent transportation and robot navigation, but it is necessary to correct the severely distorted images taken by camera with fish-eye lens into the perspective projection images that are visually perceived by human. The 3D fish-eye image correction projects the pixel points of fish-eye image into the space and then maps them into the corrected image based on the correction model. Considering the shortcomings of existing algorithms for extracting the effective region, this paper proposes an improved algorithm for such purpose to ensure both accuracy and efficiency, and presents the fish-eye image correction from the approach of 3D space.
\end{abstract}

\section{Introduction}

Nowadays, the omni-directional visual system based on fish-eye lens has been widely applied along with the rapid development of signal processing, extensive application of network transmission and improvement of computer processing speed [1]. Fish-eye lens is a camera lens with short focal length and large field of view (the angle of view ranges from $180^{\circ}$ to $270^{\circ}$ ) and gets its name as the lens looks like the bulging fish eye. The crystalline lens of human eye is oblate, allowing human to see the things further away, while the spherical crystalline lens of fish eye makes fish see only the things nearby, but have a larger angle of view. In other words, fish can see the things in a wider range [2].

For its strengths, fish-eye lens has been widely applied in all kinds of fields, but the images taken by fish-eye lens are severely distorted. If we intend to use these severely distorted images, it is necessary to correct these severely distorted images into the perspective projection images visually perceived by human [3]. At present, the correction for common cameras has been extensively studied. For instance, camera calibration corrects the camera by calculating its internal and external parameters. However, attention is rarely paid to the correction of image taken with fish-eye lens [4]. The study on the correction of distorted images taken by fish-eye camera is therefore of very high value and great significance. This paper explores the correction of distorted fish-eye images from the approach of 2D and 3D spaces, including longitudinal coordinate correction, equidistant projection correction and spherical projection constraint correction.

\section{Imaging Model of Fish-eye Image}

Fish-eye camera can be simulated with a hemisphere by taking its optic axis as the direction of shooting and its plane $O X Z$ as the imaging plane, as shown in Fig. 1. The hemispheric formula of camera is set as follows:

$$
x^{2}+y^{2}+z^{2}=r^{2}(z \geq 0, r \geq 0)
$$

Any point $P_{0}$ in the space is linked from the center to have $O P_{0}$, which intersects with the hemisphere at the point $P_{1}$. From the point $P_{1}$, a parallel line of the optic axis $O Y$ is drawn to intersect with the imaging plane $O X Z$ at the point $P_{2}$. Thus, the point $P_{2}$ is the image of the spatial 
point $P_{0}$ on the imaging plane $O X Z$. To fish-eye lens, the image of an object taken by camera is therefore a circular region [5].

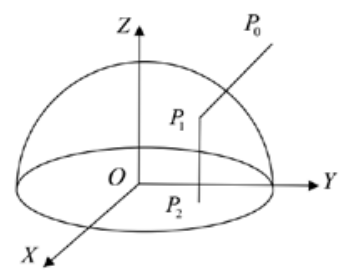

Fig. 1 Fish-eye Imaging Model

Fish-eye imaging is a kind of perspective projection, also known as linear projection. The formation process of fish-eye image can be divided into two steps as follows:

Step 1: Map every spatial point $P$ into a ray linking $P$ to the center of projection.

Step 2: Map the ray linearly into the image point. This mapping is reversible since each ray passing through the projection center $O$ corresponds to an exclusive point on the image, while each point on the image is corresponding to an exclusive ray passing through the projection center $O$. The image may be regarded to parameterize the rays passing through the projection center $O$. References present two standard methods for image parameterization. One method is to have a plane near the projection center but not passing through the center and make it intersect with the rays passing through the projection center $O$, which is the planar perspective projection model. The other method makes the center of sphere intersect with the rays passing through the projection center on the unit sphere of point, which is the spherical perspective projection model.

\section{Longitudinal Coordinate Correction of Fish-eye Image}

The extraction of circular effective region on the fish-eye image is the prerequisite for its correction. Among several common algorithms for extraction of effective region, least-square fitting algorithm is rarely applied in practice due to its troublesome computation. Area statistics algorithm may cause significant deviation when there are more black pixel points inside the effective region of fish-eye image. Line by line or column by column scan (progressive scanning) features simple and fast computation, so it is more often applied. However, this progressive scanning algorithm may fail to correct the fish-eye image if there are lots of black pixel points at any boundary of fish-eye image, since the contour edge computed in this direction moves towards the middle inevitably, turning the extracted effective region into a rectangle. Region growing algorithm performs the binary conversion of image, but this conversion entails the comparison of actual luminance with preset value for all pixels, so this algorithm will need more steps than area statistics algorithm, affecting the speed of contour extraction. This paper employs a new scanning approach to control the selection of threshold $\mathrm{T}$ and modifies the radius to enhance the accuracy and cover the shortcomings of progressive scanning and region growing algorithms, so as to guarantee both accuracy and efficiency.

\subsection{Development of Scanning Approach}

Considering the shortcomings of repeated scanning in progressive scanning, we develop a new scanning approach. As shown in Fig. 2, a rectangular coordinate system is established as the vertex at the left lower corner of fish-eye image. At the beginning of scanning, a pixel is reduced after one scanning in each direction to effectively prevent repeated scanning. If a computer scans once in each of four directions, i.e. top, bottom, left and right, the equation of scanning direction is as follows:

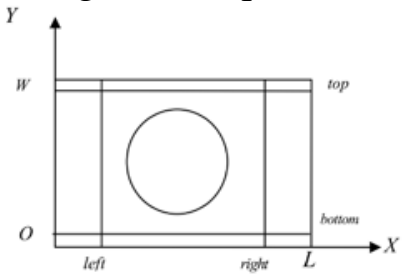

Fig. 2 Schematic Diagram of New Scanning 


$$
\left\{\begin{array}{c}
Y_{T}=W-N_{L} \\
Y_{B}=N_{B}
\end{array} \quad\left(X \in n, N_{L}<X<L-N_{R}\right)\right.
$$

Equation for vertical scanning direction:

$$
\left\{\begin{array}{c}
X_{L}=N_{L} \\
X_{R}=L-N_{r}
\end{array} \quad\left(Y \in n, N_{B}<Y<W-N_{T}\right)\right.
$$

The times of scanning needed:

$$
N=L W-4 R^{2}
$$

As shown in Fig. 2, the scanning line moves only over the region outside the square connected to the effective region.

\subsection{Selection of Control Threshold}

For progressive scanning, a key issue is the selection of control threshold. If the control threshold is too high, the extracted effective region has too small radius. If it is too low, the resistance to noise point is weaker, and the radius of extracted effective region is larger. To overcome the insufficient threshold of progressive scanning algorithm, this proposed approach conducts the binary conversion of image and compares the actual luminance with preset value for all pixel points of the binarized image. In this case, progressive scanning algorithm however needs more steps than area statistics algorithm, which affects the speed of contour extraction. This paper makes some improvements on the basis of existing algorithm to take into account both accuracy and efficiency. The steps are as follows:

Step 1: Calculate the luminance of each point scanned by the scanning line, and compare it with the threshold $T$.

Step 2: When the luminance of a pixel point scanned is larger than the threshold $T$, the scanning pauses for recording the coordinates of the point $P(x, y)$.

Step 3: Assuming that the point is scanned from left to right, the luminance of its neighboring point on the right $P(x+1, y)$ is calculated and compared with the threshold. If it is larger than $T, P(x, y)$ is the edge of effective region. If it is lower than $T$, Step 1 is repeated. It is applicable to the scanning line in any other directions. Obviously, if $P(x, y)$ is the edge of effective region, $P(x+1, y)$ locates insides the effective region (from left to right); if $P(x, y)$ is a noise point, it is rarely possible that $P(x+1, y)$ is another noise point (gray level is larger than $T$ ). Hence, this approach can strongly inhibit the noise.

\subsection{Circular Region Radius Correction of Fish-eye Image}

When progressing scanning algorithm extracts the effective region of fish-eye image, two different radii may be obtained. There are two reasons: 1 . The radii are different from each other since a great number of black pixel points exist at the circular boundary of fish-eye image; 2 . The circular region is changed into elliptic region. Assuming that $L_{1}$ and $L_{2}$ are two axes, progressive scanning algorithm cannot distinguish these two radii.

If the coordinates $X_{l}\left(x_{1}, y_{1}\right), X_{r}\left(x_{2}, y_{2}\right), X_{t}\left(x_{3}, y_{3}\right), X_{b}\left(x_{4}, y_{4}\right)$ represent the points of tangency at four edges, i.e. top, bottom, left and right, of the circular effective region on the fish-eye image, which are calculated with the progressive scanning algorithm. Under normal circumstances, the points of the coordinates $Y_{t}\left(x_{3}, y_{3}\right), Y_{b}\left(x_{4}, y_{4}\right)$ are on the same vertical line, while the points of the coordinates $X_{l}\left(x_{1}, y_{1}\right), X_{r}\left(x_{2}, y_{2}\right)$ are on the same horizontal line. Therefore, there is:

$$
\begin{gathered}
x_{3}=x_{4}=\frac{x_{1}+x_{2}}{2} \\
y_{1}=y_{2}=\frac{y_{3}+y_{4}}{2} \\
R_{1}=\frac{x_{1}-x_{2}}{2} \\
R_{2}=\frac{y_{3}-y_{4}}{2}
\end{gathered}
$$


When the effective region of fish-eye image is elliptic, formula (6) is also applicable. However, $R_{1} \neq R_{2}$, so the circular effective region of fish-eye image is distorted into elliptic, which must be corrected through multiplying by $M^{-1}$ to convert it into standard circle. The radius is corrected to $R=\max \left\{R_{1}, R_{2}\right\}$. As shown in Fig. 3, $(u, v)$ is the central point of fish-eye image, while $\alpha$ is the ratio of total line pixel points and total column pixel points on fish-eye image.

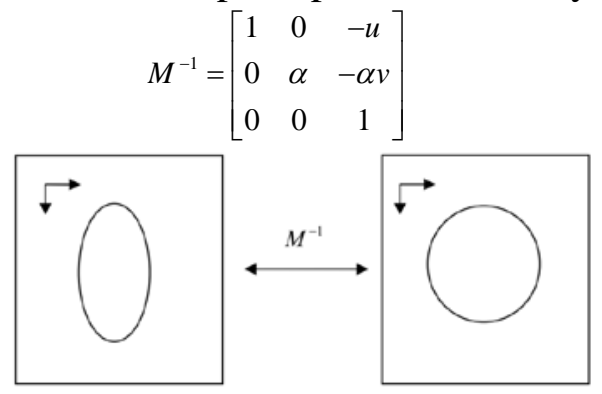

Fig. 3 Schematic Diagram of Elliptic Correction after Distortion of Effective Region

If $N, x_{1}, x_{2}, x_{3}, x_{4}$ are not suitable for formulas (5) and (6), there are lots of black pixel points at the edge of circular effective region on fish-eye image, which may be caused for two reasons: 1 . The fish-eye image is distorted, and there are black pixel points at its boundary; 2 . The fish-eye image is not distorted, but there are black pixel points at its boundary. These two reasons can be identified in the following ways:

First, mark the coordinates of top, bottom, left and right points of tangency at the boundary of fish-eye image, and judge whether the left and right points of tangency have the same vertical ordinate, and whether the top and bottom points of tangency have the same horizontal ordinate. If the horizontal ordinates of the top and bottom points are different, the black pixel points must be at the top and bottom boundaries of fish-eye image, or they must be at the left and right boundaries of fish-eye image.

Second, if two points of tangency have different horizontal or vertical ordinates, calculate the straight distance from these two points to two other points of tangency. Then, $R_{1}, R_{2}$ are calculated. If $R_{1}=R_{2}$, and there are only black pixel points at the boundaries, progressive scanning algorithm can be employed to extract the circular effective region of fish-eye image, and calculate its radius and center. If these two conditions exist simultaneously, $R=\max \left\{R_{1}, R_{2}\right\}$ is used to determine the long axis of ellipse, and take it as the radius to correct the ellipse into circle.

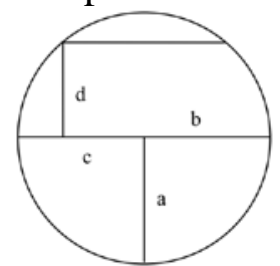

Fig. 4 Identification of Distorted Position

\section{Equidistant Projection Correction}

\subsection{Equidistant Projection}

The $Z$ axis in the camera coordinate system CCS is the optic axis of the camera. The image is formed on the $X-Y$ plane of $C C S$, and it is a circular image. In CCS , the ray towards the origin $O$ will intersect the semicircle that takes the origin as its center and has the radius of 1 . All images can be regarded to come from this sphere, and then projected into the imaging plane. It is assumed that a point $P$ in the space has the coordinates $(\theta, \phi)$ in CCS, its $\theta$ is the straight angle of $P$ and $Z$ axes, and $\phi$ is the azimuth for $P$ projection on the $X-Y$ plane. 


$$
\begin{aligned}
& p=\left(C_{x}, C_{y}, C_{z}\right)=(\sin \theta \cos \phi, \sin \theta \sin \phi, \cos \theta) \\
& \theta=a \tan 2\left(\operatorname{sqrt}\left(C_{x}{ }^{2}+C_{y}{ }^{2}\right), C_{z}\right) \\
& \phi=a \tan 2\left(C_{y}, C_{z}\right)
\end{aligned}
$$

Based on the coordinates $\left(C_{x}, C_{y}, C_{z}\right)$ of $P$ in $C C S$, the polar coordinates can be obtained as follows:

$$
\begin{aligned}
& \theta=a \cos C_{x} \\
& \phi=a \tan \left(C_{y}, C_{z}\right)
\end{aligned}
$$

The conversion between $3 D$ and $2 D$ will employ the equidistant projection model, which forms the image of $P$ on the unit hemisphere, and then projects it into a point $P$ on the unit circle. Equidistant projection means that the circular fish-eye image is a $90^{\circ}$ image with its center and sidelines evenly reflected.

$$
\begin{aligned}
& r=1 *\left(\theta / 90^{\circ}\right) \\
& x=r * \cos \phi \\
& y=r * \sin \phi
\end{aligned}
$$

In which, $r=|P O|$. For $2 D-3 D$ derivation, a point $p=(x, y)$ on the fish-eye image is given to obtain the polar coordinates of its corresponding point $P$ on the hemisphere.

$$
\begin{aligned}
& p=(\theta, \phi)=\left(r * 90^{\circ}, a \tan 2(y, x)\right) \\
& r=\sqrt{\left(x^{2}+y^{2}\right) / R}
\end{aligned}
$$

In which, $R$ is the radius.

\subsection{Calculation of Direction Angle and Elevation Angle}

Through the derivation based on the above model, it is clear that a horizon plane can be obtained based on the given visual center and the known direction of observation and angle of view. The corresponding fish-eye image on the horizon plane can be obtained through backward reasoning to obtain the elevation angle and direction angle of each point.

It is assumed that the direction of observation is vertically downward as presented in the following figure. By taking the point $P$ as the perspective center and the horizontal angle of view $\omega$, we can obtain the elevation angle and direction angle of each point on the projection plane with the width $W$ and the height $H$ through backward reasoning.

As shown in Fig. 6, the relationship between the polar coordinates $(\theta, \phi)$ and the CCS coordinates $\left(C_{x}, C_{y}, C_{z}\right)$ of the point $P$ is as follows:

$$
\begin{aligned}
& p=\left(C_{x}, C_{y}, C_{z}\right)=(\sin \theta \cos \phi, \sin \theta \sin \phi, \cos \theta) \\
& \theta=a \tan 2\left(\operatorname{sqrt}\left(C_{x}{ }^{2}+C_{y}{ }^{2}\right), C_{z}\right) \\
& \phi=a \tan 2\left(C_{y}, C_{z}\right)
\end{aligned}
$$

If $V, P, Z$ are coplanar, and $V$ is vertical to $P$ and points at $Z$ axis, there will be:

$$
d u=(2 *(r * \tan (\omega / 2)) / W)
$$

If $r$ is 1 as the radius of the circle, and it is assumed that the pixel is a square, $d v=d u$, so we can obtain the direction angle and elevation angle of each coordinate point.

Based on the above discussions, the flow chart of equidistant projection algorithm is presented in Fig. 5: 


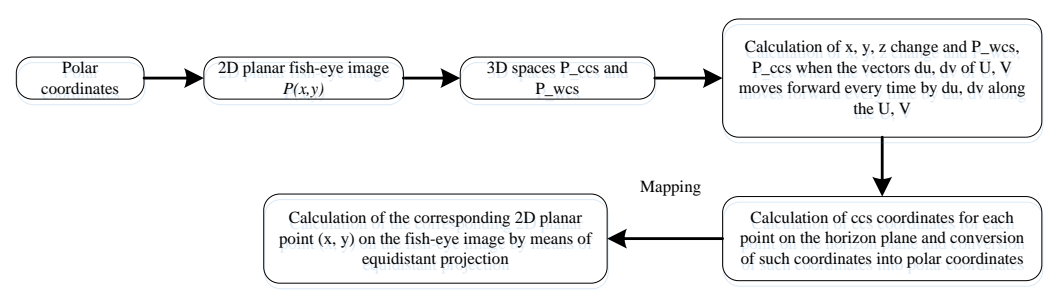

Fig. 5 Flow Chart of Equidistant Projection Correction for Fish-eye Image
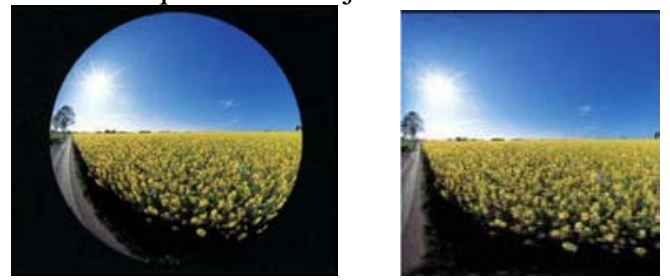

Fig. 6 Original Image and Corrected Image

This paper processes the image by taking the Intel open computer library OpenCV as an auxiliary tool. The improved algorithm for effective region is first employed to extract the circular effective region of the original fish-eye image in Fig. 6. Subsequently, equidistant correction algorithm is used to realize the correction based on the flow chart presented in Fig.6.

Table 1 presents the coordinates of center and radii obtained with these algorithms for circular ef fective region of the original image in Fig. 9, while Table 2 compares the durations of extracting the circular effective region of the original image in Fig. 9 with these algorithms.

Table 1 the coordinates of center and radii obtained with these algorithms for circular effective region of the original image

\begin{tabular}{|c|c|c|}
\hline Algorithm & Coordinates of Center & Radius \\
\hline $\begin{array}{c}\text { Progressive Scanning } \\
\text { Algorithm }\end{array}$ & $(137.5,104.5)$ & 86 \\
\hline Region Growing Algorithm & $(150.5,101.5)$ & 98.5 \\
\hline Improved Algorithm & $(1051.5,11)$ & 99 \\
\hline
\end{tabular}

Table 2 the durations of extracting the circular effective region of the original image

\begin{tabular}{|c|c|}
\hline Algorithm & Duration of Extraction (s) \\
\hline Progressive Scanning Algorithm & 0.085 \\
\hline Region Growing Algorithm & 0.096 \\
\hline Improved Algorithm & 0.080 \\
\hline
\end{tabular}

As shown in the above comparison between image correction algorithm and central region selection algorithm, the imaging spherical model with fish-eye lens is very practical and effective, and it is easy to understand and solve the model, so it is suitable for the correction of distorted fish-eye image. The proposed improved algorithm of this paper can take into account both accuracy and efficiency. The 3D fish-eye image correction algorithm performs quadric spatial transformation of pixels in the fish-eye image based on the reverse process of fish-eye imaging model, so its calculation is relatively more accurate. Normally, it employs equidistant projection. For spherical perspective projection constraint correction, it also needs to select some control points for calculation of the corresponding correction parameters, so the calculation is very troublesome. The parameters calculated in this algorithm are closely related to the type of fish-eye lens. Hence, this algorithm is often used in panoramic roaming and other techniques.

\section{Conclusion}

For short focal length and large field of view, fish-eye lens has been widely applied in such fields as virtual reality, video surveillance, intelligent transportation and robot navigation, but it is necessary to correct the severely distorted images taken by camera with fish-eye lens into the perspective projection images that are visually perceived by human. The 2D fish-eye image correction can directly map the pixel points of fish-eye image into the corrected image, while the 3D 
fish-eye image correction projects the pixel points of fish-eye image into the space and then maps them into the corrected image based on the correction model. Considering the shortcomings of existing algorithms for extracting the effective region, this paper proposes an improved algorithm for such purpose to ensure both accuracy and efficiency, and presents the fish-eye image correction from the approach of 3D space.

\section{References}

[1]Huang F, Shen X, Wang Q, et al. Correction method for fisheye image based on the virtual small-field camera. Optics Letters, 2013, 38(9):1392-4.

[2]Bi K, Huang F, Cheng W. An Image Correction Method of Fisheye Lens Based on Bilinear Interpolation. International Conference on Intelligent Computation Technology and Automation. 2011:428 - 431.

[3]Wu G, Wu. A FISHEYE IMAGE CORRECTION METHOD BASED ON BILINEAR INTERPOLATION. Computer Applications \& Software, 2012, 29(2):122-121.

[4]Zhang Q, Kamata S I. Fisheye Image Correction Based on Straight-Line Detection and Preservation. IEEE International Conference on Systems, Man, and Cybernetics. 2015.

[5]Kim H T. Fisheye Image Correction with Ellipsoid Model. 2015, 10(2):177-182. 